\title{
Analysis of Injection-locked Antenna Array
}

\author{
Kun-Chou Lee and Tah-Hsiung Chu* \\ Electrical Engineering Department, National Taiwan University \\ Taipei, Taiwan, Republic of China
}

\begin{abstract}
In this paper, analysis of dipole antenna array with each element loaded with an active device (i.e., two-terminal oscillator) is presented. The analysis is equivalent to solving a multiport network circuit with each port shunt with a twoterminal oscillator and an equivalent exciting source. A characteristic equation with the order of $2 \mathrm{~N}$ is derived to describe the stability condition of a N-element injectionlocked active antenna array. The Routh-Hurwitz stability criterion is then applied to remove the unstable solutions. Numerical results show that the array performance such as frequency locking range by taking into the array mutual coupling effect is quite different from that of an isolated antenna element.
\end{abstract}

\section{Injection-locked Oscillator}

An oscillator basically consists of three components including a feedback path, a frequency selective network, and an active device. Figure I(a) shows the circuit model of a two-terminal oscillator. As the oscillator is in the free-running state, the total admittance should be zero, i.e.,

$$
Y_{T}(V, \omega) \equiv G \tau(V, \omega)+j B_{T}(V, \omega) \equiv Y_{D}(V, \omega)+Y_{\operatorname{sel}}(\dot{\omega})=0,
$$

where $Y_{D}(V, \omega)$ is the admittance of the nonlinear active device, and $Y_{s e l}(\omega)$ is the admittance of the frequency selective network.

As the oscillator is excited by an external current source, it becomes an injectionlocked oscillator (ILO), as shown in Fig.1(b). By KCL, (1) becomes

$$
Y_{T}(V, \omega) V e^{j(\omega r+\phi)}=I_{e q} e^{j \omega i n j t} .
$$

In (2), $I_{e q}$ is the amplitude of the current source, $\omega_{i n j}$ is the frequency of injection current source, $\omega$ is the dynamic frequency, and $\phi$ is the phase difference between the voltage response and the injection current source. When the oscillator injection-locked state is achieved, the dynamic frequency $\omega$ becomes $\omega$ inj, and the output response can be determined from (2) [1].

\section{Injection-locked Active Antenna}

Consider a dipole antenna loaded with an active two-terminal device illuminated by an incident field $E_{i}$ as shown in Fig.2(a). An injection-locked active antenna (ILAA) is then constructed, and the corresponding equivalent circuit is shown in Fig.2(b), in which $Y(\omega)$ is the complex input admittance of dipole antenna, and Ieq is the amplitude of antenna short-circuit current induced by the incident field $E_{i}$. The admittance of a two-terminal active diode at the fundamental frequency can be characterized by

$$
Y_{D}(V, \omega)=\left(G_{n 0}+G_{n 2} V^{2}\right)+j \omega\left(C_{n 0}+C_{n 2} V^{2}\right),
$$

where $V$ is the amplitude of the output response. For practical semiconductor devices, $G_{n 0}$ is negative, and $G_{n 2}$ is positive. 
The KCL equation of an ILAA is the same as (2), except that $Y_{\text {sel }}(\omega)$ is replaced by the antenna input admittance $Y(\omega)$, and the exciting current source is now the short-circuit current at the antenna input terminal induced by the incident field $E_{i}$. The amplitude of the resulting terminal voltage $V$ can then be solved accordingly. There may have three possible solutions of $V^{2}$ in (2) including both stable and unstable solutions. The stability criteria of ILAA is given as [2]

$$
\begin{gathered}
2\left(G_{T} \frac{\partial_{T}}{\partial \omega}-B_{T} \frac{\partial G_{T}}{\partial \omega}\right)+\left(\frac{\partial G_{T}}{\partial T} \frac{\partial B_{T}}{\partial \omega}-\frac{\partial B_{T}}{\partial T} \frac{\partial G_{T}}{\partial \omega}\right)>0, \\
G_{T}\left(G_{T}+\frac{\partial G_{T}}{\partial \omega} V\right)+B_{T}\left(B_{T}+\frac{\partial B_{T}}{\partial V}\right)>0
\end{gathered}
$$

to remove the unstable solutions.

\section{Injection-locked Active Antenna Array}

An injection-locked active antenna array (ILAAA) is shown in Fig.3(a). Its equivalent circuit is given in Fig.3(b), where $\overline{\bar{Y}}$ denotes the antenna array input admittance matrix including the mutual coupling effects, and $l_{e q_{i}} e^{j \alpha 1}$ is the short-circuit current phasor of the $i$-th antenna element induced by the incident field $E_{i}$.

As the array injection-locked state is achieved, all the dynamic frequencies are synchronized with $\omega_{i n j}$, since all the equivalent exciting sources at the antenna input terminals are coherent. The circuit equation (2) becomes

$$
\overline{\overline{Y_{T} V}}=\overline{I_{e q}} \text {, }
$$

or,

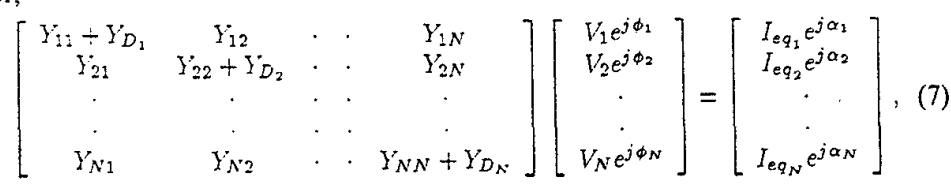

where $Y_{D i}, i=1,2, \ldots N$ is the admittance of active device at the $i$-th antenna terminal.

The derivation of stability criteria for ILAAA is similar to that of ILAA, except that the scalar variables are replaced by vector representations. The characteristic equation of an ILAAA is given as

where

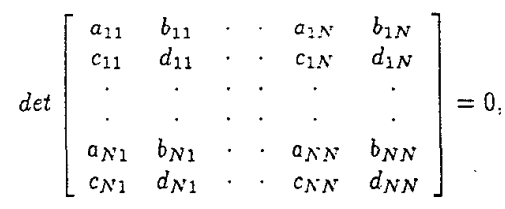

$$
\begin{aligned}
a_{i j} & =G_{T i j}+\frac{\partial G_{T i j}}{\partial V_{j}} V_{j}+S \frac{\partial B_{T i j}}{\partial \omega}, \\
b_{i j} & =-B_{T i j}+S \frac{\partial G_{T i j}}{\partial \omega}, \\
c_{i j} & =B_{T i j}+\frac{\partial B_{T i j}}{\partial V_{j}} V_{j}-S \frac{\partial G_{T i j}}{\partial \omega}, \\
d_{i j} & =G_{T i j}+S \frac{\partial B_{T i j}}{\partial \omega} .
\end{aligned}
$$


For stable solutions, all the roots of (8) must lie in the left-half $S$-plane. The KouthHurwitz stability criterion [3] can then be applied to determine the stable conditions for the solutions of (7).

\section{Numerical Examples}

In this section, numerical examples of ILAA and ILAAA are given to illustrate the analyses given above. Firstly, a single dipole antenna with length of $10 \mathrm{~cm}$ and diameter of $0.135 \mathrm{~cm}$ is considered. The two-terminal oscillator is characterized by (3) with $G_{n 0}=-0.04, G_{n 2}=0.24$. The incident wave is $E_{i}=1.0 \mathrm{~V} / \mathrm{m}$. Figure 4.4 (a) shows the resulting voltage at the antenna input terminal for different injection signal frequencies based on the ILAA analysis given in Sec.2. For convenience, all the resulting voltages and injection signal frequencies are normalized to those of the free-running case.

In the simulation of ILAAA, two parallel identical ILAA given above is considered for simplicity of analysis. The incident field is also $E_{i}=1.0 \mathrm{~V} / \mathrm{m}$. Figure $4(\mathrm{~b})$ shows the resulting voltage at each antenna input terminal for different injection signal frequencies with dipole element spacing $42.7 \mathrm{~cm}$, note $V_{1}=V_{2}=V$ due to the symmetry of the two-dipole arrangement. It shows that there is a slight change of resulting locking range due to the weak mutual coupling effect between two dipoles. As the dipole spacing is reduced to $10 \mathrm{~cm}$, the simulation result given in Fig.4(c) shows that the locking range is changed to be quite different from that of Fig.4(b). This is because the mutual coupling effect in this case is much stronger than that of Fig.4(b).

\section{Conclusion}

In this paper, the locking performance of an ILAAA is analyzed with consideration of mutual coupling effects. A characteristic equation with the order of $2 \mathrm{~N}$ is derived to describe the stable condition of a N-element ILAAA. The Routh-Hurwitz stability criterion is then applied to verify the stability property of each possible solution. Numerical results show that the array performance by taking into account the array mutual coupling effects is quite different from that of an isolated antenna element. The analysis if useful in the applications of ILAAA for communication or remote sensing.

\section{References}

[1] C.C.Huang and T.H.Chu, "Analysis of MESFET injection-locked oscillators in fundamental mode of operation," IEEE MTT-42, no. 10, pp.1851-1857, Oct. 1994.

[2] C.C.Huang and T.H.Chu, "Radiation and scattering of a slot-coupled patch antenna loaded with a MESFET oscillator". IEEE AP-43, no.3, pp.291-298, March 1995.

[3] J.V.DeVegte, Feedback Control Systems, New Jersey: Printice-Hall, 1986.

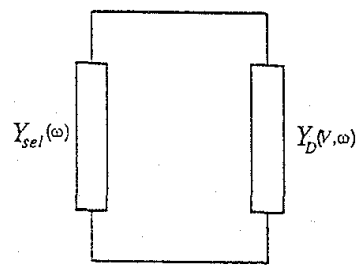

(a)

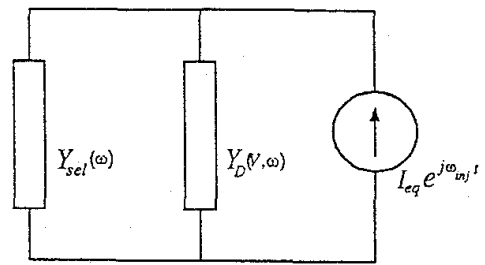

(b)

Fig.1 Equivalent circuit of (a) free-running oscillator, and (b) injection-locked oscillator. 


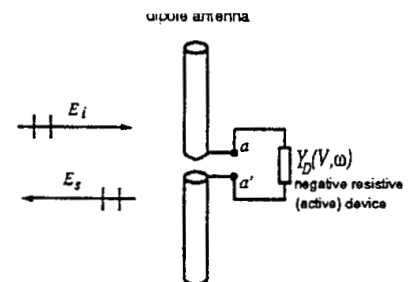

(a)

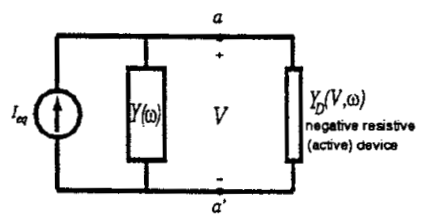

(b)

Fig.2 (a) Schernatic diagram of an injection-locked active dipole antenna, and (b) equivalent circuit.

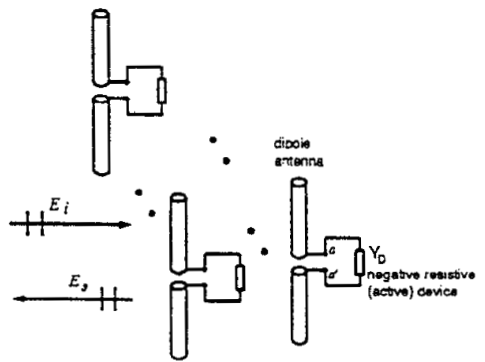

(a)

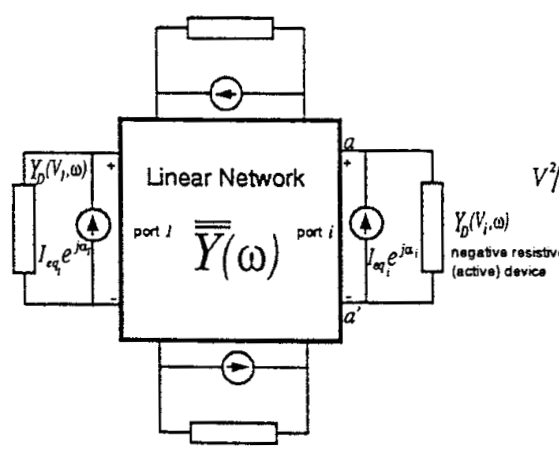

(b)

Fig.3 (a) Schematic diagram of an injection-locked active dipole antenna array, and (b) equivalent circuit.

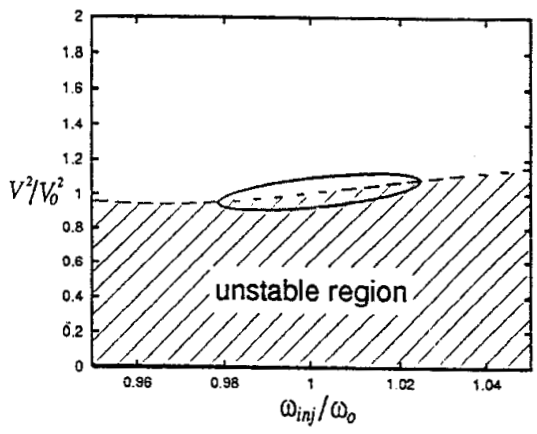

(a)

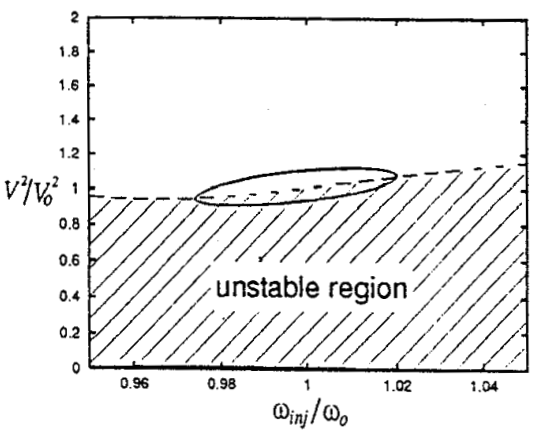

(b)

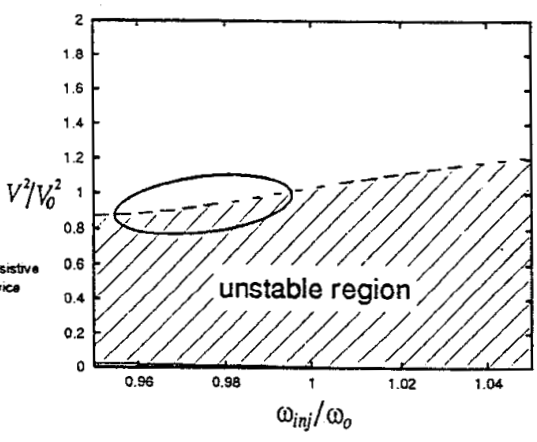

(c)

Fig. 4 Simulation resuits of the antenna input terminat voltage of (a) single ILAA, (b) two-dipole ILAAA with dipole spacing $42.7 \mathrm{~cm}$, and (c) two-dipole ILAAA with dipole spacing $10 \mathrm{~cm}$. 